\title{
Avaliação de recém nascidos pré-termo com hemorragia peri-intraventricular e/ou leucomalácia periventricular
}

\author{
Neurological evaluation of neonates with intraventricular hemorrhage and periventricular \\ leukomalacia
}

\section{Marina Junqueira Airoldi', Simone Borba do Carmo Silva², Regina Célia Turolla de Souza ${ }^{3}$}

\section{RESUMO}

Introdução. A hemorragia peri-intraventricular (HPIV) e a leucomalácia periventricular (LPV) são patologias comuns nos bebês pré-termo e estão diretamente associadas ao grau de prematuridade. Esse estudo teve como objetivo identificar alterações neurológicas em recém-nascidos pré-termo (RNPT) com HPIV e/ou LPV comparando-os com os bebês pré-termo sem estes diagnósticos. Método. Foram selecionados 19 recém-nascidos pré-termo, com menos de 32 semanas de idade gestacional, sendo 10 lactentes com diagnóstico de HPIV e/ou LPV e 9 lactentes pré-termo biologicamente normais. Os bebês foram avaliados com 40 semanas de idade gestacional corrigida utilizando-se o teste neurológico Dubowitz e Dubowitz (1970) modificado. O teste aplicado classifica os sinais neurológicos em normais, anormais ou suspeitos. Os escores do teste neurológico foram analisados e correlacionados com algumas variáveis: idade materna, tipo de parto, índice de Apgar no $5^{\circ}$ minuto, idade gestacional e peso ao nascimento. Os dados coletados foram analisados estatisticamente, considerando o índice de significância $p<0,05$. Resultados. Neste estudo, observou-se que os RNPT do grupo controle apresentaram maior índice de normalidade nas provas avaliadas $(p=0,0001)$. Constatou-se também maior número de sinais anormais nos recém-nascidos do grupo experimental ( $p=0,0002)$. Conclusão. Os bebês com diagnóstico de HPIV e/ou LPV apresentaram alterações sugestivas de déficit no comportamento motor.

Unitermos. Prematuro, Exame Neurológico, Hemorragia Intracraniana, Leucomalácia Periventricular.

Citação. Airoldi MJ, Silva SBC, Souza RCT. Avaliação de recém nascidos pré-termo com hemorragia peri-intraventricular e/ou leucomalácia periventricular.

Trabalho realizado no Hospital e Maternidade Celso Pierrô e na Maternidade de Campinas, Campinas-SP, Brasil.

1. 1.Fisioterapeuta do ambulatório de fisioterapia em neurologia infantil, Hospital de Clínicas da Universidade Estadual de Campinas, Campinas-SP, Brasil.

2. 2.Residente em neonatologia no Hospital e Maternidade Celso Pierrô, Campinas-SP, Brasil.

3. 3.Fisioterapeuta, Doutora, Docente da Pontifícia Universidade de Campinas e coordenadora do curso de fisioterapia aplicada a neurologia infantil da Unicamp, Campinas-SP, Brasil.

\section{SUMMARY}

Introduction. Hemorrhage peri-intraventricular (IPVH) and periventricular leukomalacia (PVL) are common pathologies in the premature newborn and are directly associated with the prematurity degree. The aim of this study was to evaluate, through neurological examination, premature newborns with and without intraventricular hemorrhage and periventricular leukomalacia. Method. comparative study was carried out in a population of 19 preterm neonates with less than 32 weeks of gestational age, 10 of then with intraventricular hemorrhage or periventricular leukomalacia and 9 without it. The neonates were evaluated at 40 weeks of age corrected using the neurological tests proposed by Dubowitz and Dubowitz (1970) modified. The evaluations were classified as normal, abnormal or suspected. The neurological test had been analyzed and correlated with some variable as: mother's age, gestational age, weight and Apgar score. The collected data had been analyzed through statistical tests with $p$ values less than 0.05 . Results. In this study, it was observed that the premature newborn of the group controls had presented greater itens of normality in the evaluated tests $(p=0.0001)$. A greater number of abnormal signs were found in the neonates with intraventricular hemorrhage and periventricular leukomalacia $(p=0.0002)$.Conclusion. In conclusion, most neonates with IPVH or PVL showed a significant correlation between the clinical and laboratory findings with neurological evaluation.

Keywords. Infant Premature, Neurological Examination, Intracranial Hemorrhages, Leukomalacia Periventricular Citation. Airoldi MJ, Silva SBC, Souza RCT. Neurological evaluation of neonates with intraventricular hemorrhage and periventricular leukomalacia.

Endereço para correspondência: Ambulatório de Fisioterapia aplicada a Neurologia Infantil Hospital de Clínicas da Universidade Estadual de Campinas Serviço de Fisioterapia e Terapia Ocupacional Cidade Universitária Zeferino Vaz $\mathrm{s} / \mathrm{n}^{\circ}, 3^{\circ}$ andar CEP 13085-970, Campinas-SP, Brasil. Tel: 1935217374

E-mail:majufisio@yahoo.com.br

Recebido em: 15/01/2009 Revisado em: 16/01/2009 a 17/03/2009

Aceito em: 18/03/2009

Conflito de interesses: não 


\section{INTRODUÇÃO}

Nas últimas décadas tem-se aumentado o interesse científico referente ao desenvolvimento de bebês pré-termo. Um dos marcos de atenção a essa população foi o avanço das ciências médicas juntamente com a implantação das modernas unidades de terapia intensiva neonatais equipadas com tecnologia de ponta que reduzem a mortalidade destes bebês. Por outro lado, o cuidado presente nestes lugares altamente qualificados e especializados, tem contribuído com o aparecimento de iatrogênias no processo de crescimento e desenvolvimento destas crianças ${ }^{1}$.

$\mathrm{O}$ aumento da incidência de paralisia cerebral na década de 80 coincidiu com o aumento de sobrevida dos prematuros. Uma das explicações para este fato reflete a sobrevivência de bebês submetidos intra-uterinamente a um insulto isquêmico, resultando em nascimento prematuro e lesão da substância branca que mais tarde pode se manifestar como paralisia cerebral. Entretanto, este fato pode ser resultado da sobrevivência de maior proporção de bebês imaturos que são particularmente mais vulneráveis a processos isquêmicos e hemorrágicos no período intraparto e neonatal. Uma terceira possibilidade é de que a paralisia cerebral represente o ponto final de uma cadeia de efeitos adversos, ocorrendo em um período em que o cérebro esta particularmente vulnerável a isquemia ${ }^{2}$.

A maior sobrevida de recém nascidos prétermo determinou maior risco de problemas neurológicos relacionados a hemorragias intracranianas $^{3}$. A hemorragia peri-intraventricular é comum no período neonatal e a sua fisiopatologia está intimamente ligada à matriz germinativa, que é considerada o principal local de origem do processo hemorrágico. A matriz germinativa dá origem aos neuroblastos ou células germinais do cérebro, entre a $10^{\text {a }}$ e a $20^{\text {a }}$ semana de idade gestacional, e aos glioblastos no terceiro trimestre, que em seguida se diferenciam em astrócitos e oligodendrócitos. Ao final do $2^{\circ}$ trimestre da gravidez, a matriz germinativa é uma região com alta atividade metabólica e a parede endotelial nessa área ainda está imatura e frágil. Essas vulnerabilidades anatômicas, associadas à imaturidade de auto-regulação vascular nessa época, contribuem para a suscetibilidade da matriz germinativa à hemorragia em resposta a flutuações na pressão cardiovascular ${ }^{4,5}$
A hemorragia peri-intraventricular (HPIV) é classificada de acordo com a sua magnitude. $\mathrm{O}$ sangramento pode ficar restrito à matriz germinativa ou romper a parede do ventrículo lateral subjacente, inundando-o de sangue. É classificada como grau I quando a hemorragia subependimária fica confinada somente à matriz germinativa; grau II quando ocorre a hemorragia intraventricular sem dilatação ventricular (enchimento de sangue a menos de $50 \%$ do ventrículo na cintigrafia parassagital); grau III quando ocorre a hemorragia intraventricular com dilatação ventricular; e grau IV quando ocorre o infarto hemorrágico periventricular ${ }^{6}$.

A leucomalácia periventricular (LPV) também é bastante comum em bebês pré-termo e refere-se a áreas de necrose celular da substância branca em localização específica do cérebro fetal, adjacente aos ângulos externos dos ventrículos laterais ${ }^{7}$.

O diagnóstico depende da ultra-sonografia cerebral, que revela uma evolução típica, mostrando inicialmente áreas de ecogenicidade aumentada na região periventricular. Depois de alguns dias, forma-se cavitação nessas áreas ecodensas que evoluem para pequenos cistos localizados representando os focos necróticos geralmente na região frontoparietal ou na substância branca occipital e frontoparieta para lesões císticas extensas1. Os cistos são diagnosticados quando superiores a $0,5 \mathrm{~cm}$ de diâmetro. Esse processo de formação cística ocorre no decorrer de semanas, de modo que a identificação e a classificação da LPV depende do momento em que estudos ultrassonográficos são feitos na criança, bem como da quantidade ${ }^{4,8}$.

O exame patológico mostra redução da mielina. A fisiopatologia está bem esclarecida, sendo a leucomalácia uma lesão da zona marginal. $\mathrm{O}$ fator principal de risco é um decréscimo do fluxo sanguíneo cerebral. Esse tipo de isquemia da zona marginal pode ocorrer após hipotensão, bradicardia ou parada cardíaca?.

O sistema nervoso do neonato é dinâmico e tem rápido desenvolvimento. Todos os exames devem se atentar não somente a possibilidade de alguma patologia como também ao estado de maturidade do sistema nervoso. A tentativa de se dar o prognóstico deve considerar o enorme potencial de resolução e compensação do desenvolvimento do sistema nervoso, que provavelmente é proporcional a sua imaturidade ${ }^{10}$. 
O exame neurológico permite o diagnóstico precoce das lesões cerebrais e déficits motores, avaliando a integridade do sistema nervoso central e possibilitando a intervenção precoce. A literatura aponta o exame neurológico de Dubowitz e Dubowitz (1970) como ferramenta investigativa, de confiabilidade e validade ${ }^{10}$. O exame, escolhido para ser aplicado neste estudo, é pratico e objetivo, podendo ser aplicado em 15 minutos e sua aplicação pode ser feita nos primeiros dias de vida do bebê, além de poder ser feito dentro das incubadoras. O exame é composto por 33 itens neurológicos e neurocomportamentais que são classificados em normais, suspeitos ou anormais, identificando precocemente as incapacidades neurológicas em crianças de alto risco.

Este estudo teve como objetivo identificar alterações neurológicas em recém- nascidos prétermo com hemorragia peri-intraventricular e/ou leucomalácia periventricular e comparar os escores do exame neurológico destes recém-nascidos com bebês pré-termo sem este diagnóstico. Também se buscou relacionar as variáveis, como idade gestacional, peso, Apgar e idade materna com a presença de diagnóstico de hemorragia Peri-intraventricular e/ou leucomalácia periventricular.

\section{MÉTODO}

\section{Amostra}

Foram avaliados 19 recém-nascidos prétermo (RNPT) (12 do sexo feminino e 7 do sexo masculino), divididos em dois grupos: 1) Grupo experimental (GE), incluindo 10 sujeitos com o diagnóstico de leucomalácia periventricular e/ou hemorragia peri-intraventricular; 2) Grupo controle (GC), incluindo 9 sujeitos sem este diagnóstico para comparação através do exame neurológico Dubowitz e Dubowitz (1970) modificado10. Todos os RNPT foram selecionados na Maternidade de Campinas, nascidos entre 25 a 32 semanas de idade gestacional. Todos os responsáveis pelos RNPT assinaram o termo de Consentimento Livre e Esclarecido. Este estudo foi aprovado pelo Comitê de Ética da Universidade Católica de Campinas com protocolo 605/05 e recebeu apoio da Maternidade de Campinas.

Foram excluídos desse estudo os RNPT com má-formação congênita, síndrome genética associada, que tenham tido outro acometimento do sistema nervoso central, que apresentaram condi- ção clínica desfavorável à avaliação, e bebês que evoluírem para óbito durante o estudo.

\section{Procedimento}

Aplicou-se um questionário de anamnese para a caracterização da amostra e para a verificação de dados como: idade gestacional (segundo Capurro) ${ }^{11}$, peso ao nascimento, índice de Apgar, tipo de parto e confirmação do diagnóstico de HPIV e/ou LPV assim como o seu grau de comprometimento (diagnosticado através da ultra-sonografia cerebral).

Como instrumento específico de estudo foi realizado o exame neurológico Dubowitz e Dubowitz (1970)10, e foram avaliados 23 itens, sendo eles: postura e tono; retorno a flexão do antebraço; tração dos membros superiores direito e esquerdo; retorno a flexão de membros inferiores; tração de membros inferiores direito e esquerdo; ângulo poplíteo direito e esquerdo; controle de cabeça 1; controle de cabeça 2; queda da cabeça; suspensão ventral; reflexo de sucção; preensão palmar, preensão plantar; colocação plantar; reflexo de moro; tono flexor 1; tono flexor 2; tono extensor da perna; tono extensor de pescoço; e tono extensor.

Os itens são registrados seqüencialmente em uma mesma folha e contém um diagrama ao lado de cada item para facilitar sua aplicação ${ }^{10}$.

Todos os RNPT foram avaliados com 40 semanas de idade gestacional corrigida. A correção da idade gestacional permite isolar as diferentes respostas inerentes aos vários estágios de maturação abaixo de 40 semanas e é calculada subtraindo-se da idade cronológica o número de semanas que faltavam ao nascer para o bebê alcançarem o termo (40 semanas). (Idade corrigida = idade cronológica - (40 semanas - idade gestacional ao nascimento, em semanas).

\section{Análise Estatística}

Para análise estatística deste estudo, utilizou-se o teste de Mann-Whitney para análise de freqüência e de score na comparação de duas amostras não paramétrica. Utilizou-se o teste $t$ de Student não pareado, para amostras independentes com distribuição normal. O teste estatístico de Kruskal-Wallis foi utilizado para análise de 3 amostras independentes. O nível de significância adotado para os testes estatísticos foi de 0,05. Também foram realizados cálculos simples de porcentagem e tabelas descritivas. 


\section{RESULTADOS}

No Grupo Experimental (GE) foram avaliados 10 RNPT, sendo 3 com diagnóstico de LPV Bilateral, 4 com HPIV Grau I, 2 com HPIV Grau II e 1 com HPIV Grau I e II. Este grupo teve como valores de média de peso $940 \mathrm{~g}$, idade gestacional de 28 semanas, Apgar no $1^{\circ}$ minuto de 5,4, Apgar $5^{\circ}$ minuto de 7,$7 ; 70 \%$ dos RNPT eram do sexo feminino e a idade materna variou entre 21 a 44 anos. O Grupo controle teve valores de média de peso de $1511 \mathrm{~g}$, idade gestacional de 30 semanas, Apgar no $1^{\circ} \mathrm{mi}-$ nuto de 6,5 , Apgar no $5^{\circ}$ minuto de 8,$7 ; 55,5 \%$ dos RNPT eram do sexo feminino e a idade materna variou entre 19 a 40 anos.

O grupo estudo apresentou escores menores para os itens: tração em membros superiores direito e esquerdo, retorno a flexão de membros inferiores, tração em membros superiores esquerdo e direito, controle de cabeça 1 e 2, queda de cabeça, suspensão ventral, colocação plantar e reflexo de moro, analisados na avaliação de Dubowitz e Dubowitz (Tabela 1). É importante ressaltar que para cada item analisado, os autores estipulam escores de acordo com o desempenho motor do bebê de 0; 0,5; ou 1 que recebem a classificação de anormal, suspeito ou normal respectivamente.

Os RNPT com diagnóstico de HPIV e/ou LPV mostraram menor quantidade de itens normais ( $p=0,0001$, Gráfico 1) e maior quantidade de itens anormais ( $p=0,0002$, Gráfico 2) quando comparados a RNPT sem este diagnóstico; tal resultado, demonstra maior desempenho e melhor condição motora dos bebês sem diagnóstico de HPIV e/ou LPV.

Considerando a idade gestacional segundo Capurro 11 pode se observar que os RNPT que nascem com menor idade gestacional têm maior chance de desenvolver HPIV e/ou LPV ( $p=0,0275$, Gráfico 3).

Os valores de peso ao nascimento dos RNPT com diagnóstico de HPIV e/ou LPV foram menores que os valores dos RNPT sem este diagnóstico $(p=0,0001$, Gráfico 4).

\section{DISCUSSÃO}

A avaliação é um processo contínuo de coleta e organização de informações relevantes para planejar e implementar um trabalho efetivo. Instrumentos de avaliação do lactente têm como objetivos conhecer as características de normalidade de determinada população ou grupo, promover intervenções precoces nos desvios do desenvolvimento, recolher informações sobre o nível de operação ou marcos alcançados, revelar a velocidade e direção do desenvolvimento e avaliar os testes assegurando a confiabilidade e validade ${ }^{12}$.

Tabela 1. Mediana dos valores referentes aos itens avaliados do Grupo Estudo (GE) e do Grupo Controle (GC).

\begin{tabular}{|l|l|l|l|}
\hline itens avaliados & GE & GC & valor de $p$ \\
\hline Postura e Tono & 0,35 & 0,66 & \\
\hline Retorno a flexão MS & 0,20 & 0,66 & \\
\hline Tração MSD & 0,20 & 0,77 & $*$ \\
\hline Tração MSE & 0,00 & 0,87 & $* *$ \\
\hline Retorno a flexão MI & 0,20 & 0,77 & $*$ \\
\hline Tração MID & 0,45 & 0,88 & $*$ \\
\hline Tração MIE & 0,25 & 1,00 & $* *$ \\
\hline Ângulo Poplteo D & 0,90 & 0,93 & \\
\hline Ângulo Popliteo E & 0,60 & 0,88 & \\
\hline Controle Cabeça (1) & 0,50 & 0,88 & $*$ \\
\hline Controle Cabeça (2) & 0,30 & 0,55 & $*$ \\
\hline Queda da cabeça & 0,20 & 0,50 & $*$ \\
\hline Suspensão Ventral & 0,23 & 0,79 & $*$ \\
\hline Sucção & 0,53 & 0,79 & \\
\hline Preensão Palmar & 0,61 & 1,00 & \\
\hline Preensão Plantar & 0,66 & 0,66 & \\
\hline Colocação Plantar & 0,38 & 1,00 & $* *$ \\
\hline Moro & 0,21 & 0,70 & $* *$ \\
\hline Tono flexor 1 & 1,00 & 100 & \\
\hline Tono flexor 2 & 0,90 & 1,00 & \\
\hline Tono extensor MI & 0,53 & 1,00 & \\
\hline Tono extensor pescoço & 0,25 & 0,35 & \\
\hline MS mem & * & \\
\hline
\end{tabular}

MS=membro superior; $M I=$ membro inferior; $D=$ direito; $E=$ esquerdo; $* \mathrm{p} \leq 0,05 ; * * \mathrm{p} \leq 0,01$.

No presente estudo, os RNPT do GC tiveram escore maior do que o $\mathrm{GE}$, em todos os itens de avaliação utilizados neste trabalho, sendo estatisticamente significantes os itens de tração de membro superior direito, tração do membro superior esquerdo, retorno a flexão de membro inferior, tração membro inferior esquerdo, tração de membro inferior direito, controle de cabeça 1, controle de cabeça 2, queda da cabeça, suspensão ventral, colocação plantar e reflexo de Moro.

É interessante ressaltar que no período de coleta de dados deste estudo, não nascera RNPT com HPIV grau III e IV, portanto, os RNPT do GE apresentaram o diagnóstico de HPIV grau I e grau II. De acordo com a literatura, a evolução neurológica de crianças com HPIV grau I ou II é tida em 
geral como favorável, e a maioria dos bebês não apresentam seqüelas. Por outro lado, em estudo onde se comparou crianças com HPIV e crianças sem esse diagnóstico, as crianças com comprometimentos leves, grau I e II, tiveram o risco de $11 \%$ de ter alguma seqüela neurológica ${ }^{13}$.

Gráfico 1. Comparação da quantidade de itens normais de recém-nascidos pré-termo do grupo estudo e do grupo controle.

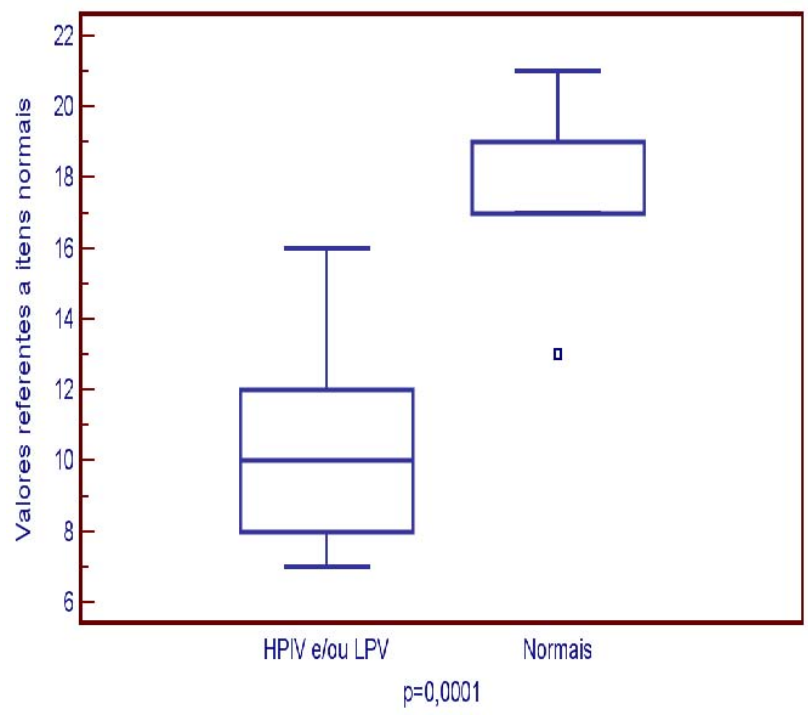

Gráfico 2. Comparação da quantidade de itens anormais de recém-nascidos pré-termo do grupo estudo e do grupo controle.

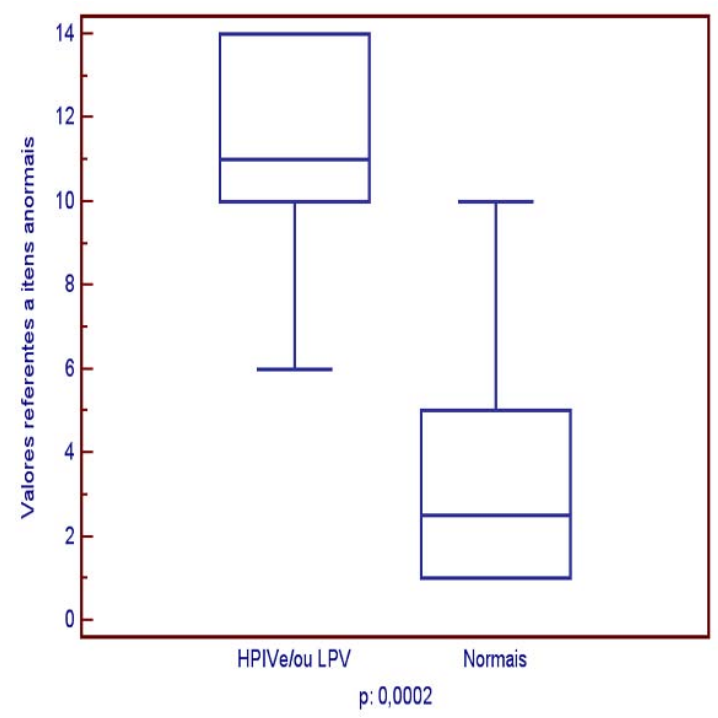

Pode-se perceber déficit motor das crianças com HPIV grau I e grau II que compuseram o GE juntamente com os RNPT com LPV. Encontrou-se melhor desempenho do GC que teve média de 19 itens normais na sua avaliação enquanto que o GE obteve a média de 10 itens normais na sua avaliação.

A análise dos itens anormais observados na avaliação também demonstram melhor desempenho motor do GC que teve média de 3 itens anormais enquanto que o GE apresentou média de 11 itens anormais.

Gráfico 3. Comparação de valores da idade gestacional (IG) em semanas, segundo índice de Capurro ${ }^{11}$ de recém-nascidos pré-termo do grupo estudo e grupo controle.

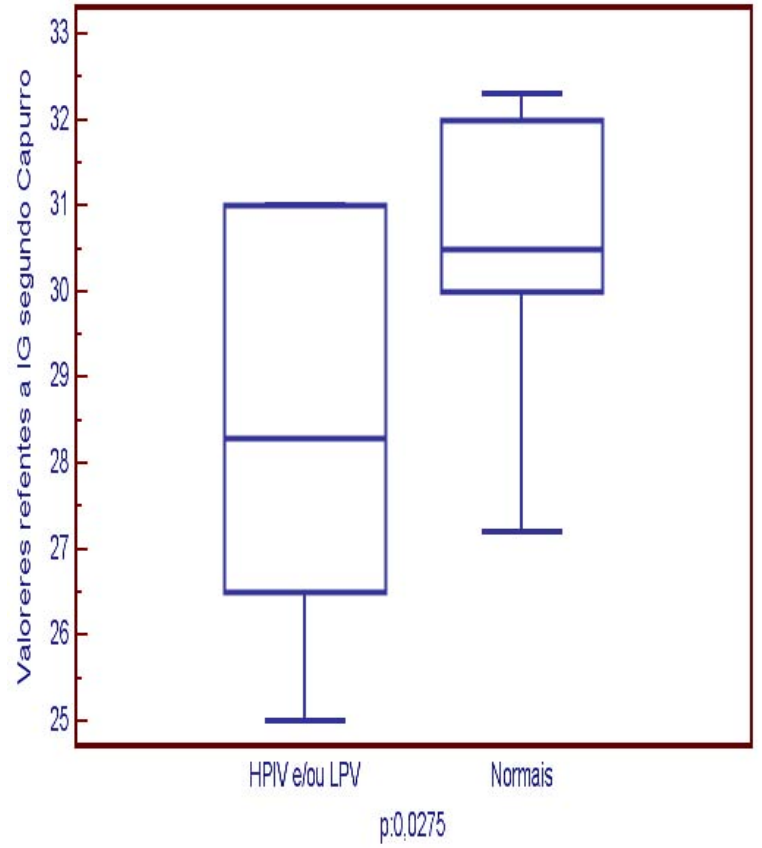

Gráfico 4. Comparação de valores do peso (em gramas) ao nascimento de recém-nascidos pré-termo do grupo estudo e grupo controle.

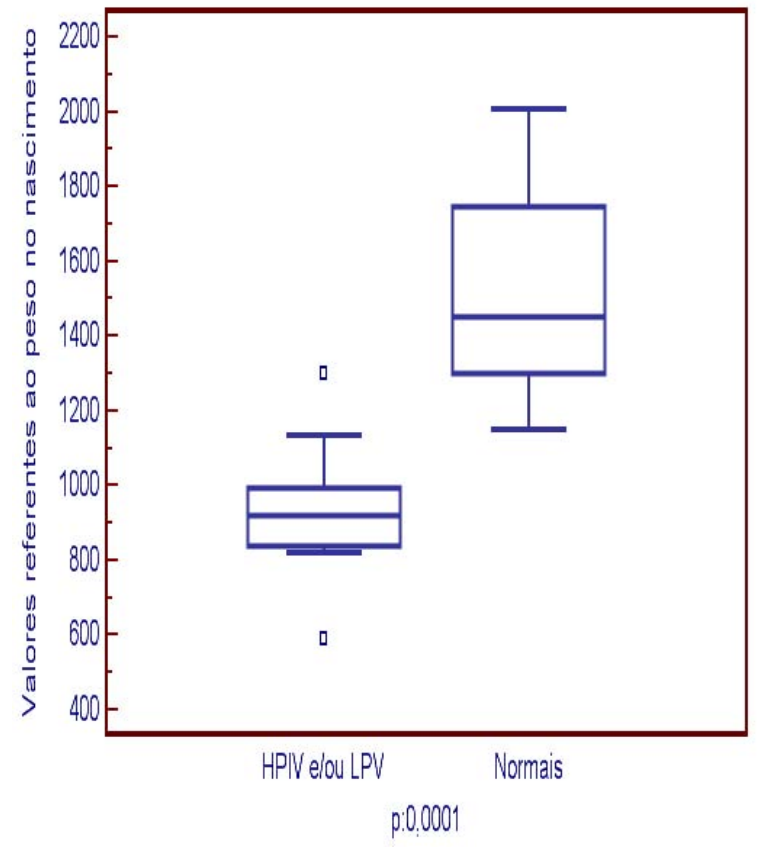


Em estudo com 65 RNPT com HPIV e 65 RNPT sem este diagnóstico, avaliados pelo exame neurológico Dubowitz e Dubowitz, também encontrou maior número de sinais anormais nos recém-nascidos com HPIV2. De acordo com a literatura, quanto maior o número de sinais desviantes, maior a probabilidade de anormalidades futuras ${ }^{2,10}$.

Pesquisas têm demonstrado que quanto mais precoce é o nascimento do bebê, maior é a influência sob a anatomia e a biodinâmica desta criança, podendo interferir no seu desenvolvimento motor ${ }^{14-16}$.

Neste estudo encontrou-se relação direta entre a idade gestacional e o diagnóstico de HPIV e/ou LPV. A média de idade gestacional do grupo controle foi de 30,5 semanas e a média do grupo experimental de 28 semanas.

Outros autores também demonstram que a incidência de HPIV varia de acordo com o grau de prematuridade, com incidência em torno de $20 \%$ em RNPT com idades gestacionais entre 30 e 35 semanas aumentando para valores que variam de 3 $0 \%$ a $75 \%$ em bebês com idades gestacionais abaixo de 30 semanas $^{15}$.

Em estudo sobre incidência, foi encontrado $61 \%$ de HPIV em RNPT com menos de $1.000 \mathrm{~g}$, mostrando também que o baixo peso é um fator de risco bastante importante para o diagnóstico de HPIV17.

Avaliou-se 100 bebês com extremo baixo peso com idade corrigida de 1 ano, e constatou-se que $51 \%$ das crianças apresentavam exames neurológicos anormais ou suspeitos e dentro deste grupo $24 \%$ tiveram o diagnóstico de PC, demonstrando que bebês de extremo baixo peso estão mais suscetíveis para desenvolverem atrasos significativos no desenvolvimento ${ }^{18}$.

No estudo presente, o peso dos RNPT também foi um fator relacionado ao diagnóstico de HPIV e/ou LPV. A média dos RNPT do grupo controle foi $1.500 \mathrm{~g}$., enquanto a média dos RNPT do grupo experimental foi $900 \mathrm{~g}$.

\section{CONCLUSÃO}

Pôde-se observar que o exame neurológico de Dubowitz e Dubowitz (1970) foi adequado para avaliação dessa amostra. Os RNPT com diagnóstico de HPIV e/ou LPV tiveram em sua avaliação mais itens classificados como anormais, sendo tais alterações sugestivas de déficits no comportamento neuro- motor. Já os RNPT sem o diagnóstico de HPIV e/ou LPV tiveram maior pontuação nos itens classificados como normais, comprovando que estes bebês estão menos propícios a déficits neurológicos.

$\mathrm{Na}$ análise das variáveis, foi significativa a diferença encontrada entre os dois grupos na análise da idade gestacional e do peso demonstrando que tais valores são inversamente proporcionais ao aparecimento das patologias confirmando o que existe na literatura.

\section{REFERÊNCIAS BIBLIOGRÁFICAS}

1.Rugolo LMSS. Crescimento e desenvolvimento em longo prazo do prematuro extremo. J Pediatr 2005;81(1):1-17.

2.Stopiglia MCS, Ribeiro MVLM, Marba S. Neurological evaluation of neonates with intraventricular and periventricular hemorrhage. Arq Neuropsiquiatr 1999;57(2):366-70.

3.Luu TM, Ment LR, Scheneider KC, Katz KH, Allan WC, Vohr BR. Lastin effects of preterm birth and neonatal brain hemorrhage at 12 Years of Age. Pediatrics 2009;123(3):1037-44.

4.Sweeney JK, Swanson MW. Crianças de Baixo Peso ao Nascer: Cuidados Neonatais e Acompanhamento. In: Umphred DA. Reabilitação Neurológica. Barueri: Manole, 2004, 217-20.

5.Carvalho ES, Carvalho WB. Terapêutica e Prática Pediátrica. 2.ed. São Paulo: Atheneu, 2000, 1178.

6.Volpe JJ. Neurology of the Newborn. 4.ed, Philadelphia: WB Saunders Company, 2001, 430.

7.Blumenthal I. Periventricular leucomalacia: a review. Eur J Pediatr 2004;163:435-42. 8.Silvera RC, Procianoy RS. Lesões isquêmicas cerebrais no recém-nascido pré-termo de muito baixo peso. J Pediatr 2005;81(1):330-8.

9.Fanaroff KE Alto risco em Neonatologia. 4.ed. Rio de Janeiro: Guanabara Koogan, 1995, 66.

10.Dubowitz LMS, Dubowitz V. The neurological assessment of the preterm and full-term newborn infant. Clin Dev Med 1981;79(1):1038.

11.Capurro H, Konichezky S, Fonseca D. A Simplified method for diagnosis of gestacional age in the newborn infant. J.Pediatr 1978;93:1202.

12.Silva PL. Influência de práticas maternas no desenvolvimento motor de lactentes do $6^{\circ}$ ao $12^{\circ}$ meses de vida (Dissertação) Piracicaba: Universidade Metodista de Piracicaba, 2005, 124p.

13.Marba STM. Hemorragia Peri-intraventricular: Incidência em recém-nascidos vivos e sua associação com idade gestacional, peso, crescimento intra-uterino e óbito neonatal (Dissertação) Campinas: Faculdade de Ciências Médicas da Universidade Estadual de Campinas, 1993, 99p.

14.Gaetan ESM, Moura-Ribeiro M V. Developmental study of early posture control in preterm and fullterm infants. Arq Neuropsiquiatr 2002;60(4):954-8.

15.Morales OLS, Latorre JFL, Hernandez JR, Vera LAP. Hemorragia intraventricular em ninos prétermino, incidência y factores de riesgo. Med Unab 2003;6(17):57-62.

16.Silveira RC, Procianoy RS, Dill JC, Costa CS. Sepse neonatal como fator de risco para leucomalácia periventricular em pré-termos de muito baixo peso. J Pediatr 2008;84(3):211-6.

17.Mancini MC, Barbosa NL, Banwart D, Silveira S, Guerpelli JL, Leone $C R$. Intraventricular hemorrhage in very low birth weight infants: associated risk factors and outcome in the neonatal period. Rev Hosp Clin FMUSP 1999;54(5):151-4.

18.Tecklin JS. Fisioterapia Pediátrica. 3.ed. Porto Alegre: Art Med, 2002, 69-101. 